\title{
LEAP-FROG METHOD FOR STOCHASTIC FUNCTIONAL WAVE EQUATIONS*
}

\author{
HENRYK LESZCZYŃSKI ${ }^{\dagger}$, MILENA MATUSIK $^{\dagger}$, AND MONIKA WRZOSEK ${ }^{\dagger}$
}

\begin{abstract}
We perform a time-space discretisation, known as the leap-frog method, for nonlinear stochastic functional wave equations driven by multiplicative time-space white noise. To prove its stability we apply Cairoli's maximal inequalities for two-parameter martingales and provide a lemma for estimating solutions to a class of stochastic wave equations and a Gronwall-type inequality over cones. The method converges in $L^{2}$ at a rate of $O(\sqrt{h})$, where $h$ is a time-space step size.
\end{abstract}

Key words. leap-frog, stochastic wave equation

AMS subject classifications. 60H15, 35R60, 35R 10

1. Introduction. We are interested in applying the leap-frog (LF) method to the initial value problem for the nonlinear stochastic wave equation (SWE) with a non-local dependence

$$
\left\{\begin{aligned}
\frac{\partial^{2} u}{\partial t^{2}}-\frac{\partial^{2} u}{\partial x^{2}} & =f\left(t, x,\left.u\right|_{C_{t, x}}\right)+g\left(t, x,\left.u\right|_{C_{t, x}}\right) \dot{W} & & \text { for }(t, x) \in \mathbb{R}_{+} \times \mathbb{R}, \\
u(0, x) & =\phi(x), \quad \frac{\partial u}{\partial t}(0, x)=\psi(x) & & \text { for } x \in \mathbb{R},
\end{aligned}\right.
$$

where $\dot{W}$ is time-space white noise. The LF method is known by different names in the literature, e.g., the Störmer method and the Encke method (in astronomy); see the review article [7]. In molecular dynamics it is often referred to as the Verlet method. In the context of partial differential equations (PDE) of wave propagation, the most common term is leap-frog. The method is two-step, explicit, second-order accurate, and simple to implement. It has some important geometric properties and preserves the wave energy conservation as it is symplectic [10]. Hence, it is widely used in the calculation of the atmosphere, oceans, and computer graphics [21]. In [5] the authors propose a two-level conservative modification of the classical LF scheme which is second-order accurate on nonuniform grids and can be used for solving fluctuating hydrodynamics problems. In [18] a LF scheme for the optimal control of parabolic PDE is shown to be unconditionally stable and second-order convergent without the requirement of the classical Courant-Friedrichs-Lewy condition on the spatial and temporal mesh step sizes. LF schemes are applied to Maxwell equations in [17, 19]. The implicit-explicit combination of Crank-Nicolson and LF schemes, frequently used in atmosphere, ocean, and climate codes, is studied in [11]. Some extensions of LF methods are presented in [27].

In the 1960's, wave equations subject to random perturbations have attracted a lot of attention due to their applications in physics, relativistic quantum mechanics, and oceanography. Let us mention two interesting examples presented in [4]. DNA molecules can be seen as long elastic strings modeled by a wave equation. While floating in the liquid, they are exposed to impacts of the fluid's molecules, which can be described as a stochastic force. These phenomena are of biological interest; in particular: when a DNA strand moves around and two normally distant parts of the string get close enough together, it can happen that a biological event occurs: for instance, an enzyme may be released; cf. [4]. Some recent studies show that a DNA molecule acts like a receiver or transmitter of radio waves communicating

${ }^{*}$ Received May 21, 2019. Accepted November 11, 2019. Published online on February 3, 2020. Recommended by Kevin Burrage. Supported by grant BMN 539-5100-B349-19 from the University of Gdańsk.

${ }^{\dagger}$ University of Gdańsk, Wita Stwosza 57, 80-952 Gdańsk

(\{hleszcz, mnetka, mwrzosek\}@mat.ug.edu.pl). 


\section{ETNA}

Kent State University and

Johann Radon Institute (RICAM)

with other DNA molecules or other molecules. This property can be used to prevent the progression of some diseases like cancer [26]. For a deeper discussion of DNA interactions, we refer the reader to $[1,6]$. Another example concerns a motion of the sun's surface, which can provide information about the internal structure of the sun [9]. Solar geophysicists are especially interested in determining the origin of shock waves going around the surface, and these shocks can be modeled in a probabilistic sense.

Numerical methods applied to SWEs include the method of lines [24], finite element approximations [15], semi-analytic methods based on Fourier series expansions [25], stochastic trigonometric schemes for the temporal approximations [3], spectral Galerkin methods for spatial approximations and exponential time integrators for temporal approximation [31], Newton's method [16], and others [20].

In [8] it is shown that under some conditions, the accuracy of the LF approximation of the solution to a quasi-linear SWE driven by time-space white noise is of weak order two, i.e.,

$$
\left|\mathbb{E}\left[\phi\left(\hat{u}_{h}\right)\right]-\mathbb{E}[\phi(u)]\right| \leq C h^{2} .
$$

Upon the space discretisation, the author performs a change of coordinates resulting in SWEs with inhomogeneous boundary conditions. The study is conducted in the semigroup framework [22], while in [29] the variational approach is used to investigate the convergence of LF schemes for SWEs in $L^{p}, p \geq 1$. It is shown that the optimal rate of convergence is $O(\sqrt{h})$ and $O\left(\sqrt{h \mid \log h^{\epsilon}}\right)$ on compact sets for $\epsilon>0$ and a time-space step size $h$. The proof requires formulating a series of lemmas, e.g., a $2 p$-th moment inequality and a $p$-th moment maximal Cairoli-type inequality for stochastic Itô integrals over cones for $p>1$.

Our purpose is to extend the results obtained by Walsh [29] to the case of a SWE with a non-local dependence, e.g.,

$$
g\left(t, x,\left.u\right|_{C_{t, x}}\right)=\sin \left(\int_{C_{t, x}} u(s, y) d y d s\right) .
$$

Taking $f\left(t, x,\left.u\right|_{C_{t, x}}\right)=f(t, x, u)$ and $g\left(t, x,\left.u\right|_{C_{t, x}}\right)=g(t, x, u)$, one arrives at the problem discussed in [29]. We establish the stability of the LF scheme for the SWE, which by virtue of the Lax theorem is equivalent to convergence assuming the consistency of the scheme.

The paper is organized as follows. In Section 2 we set up notation and formulate the problem. Section 3 contains details of the LF scheme. In the fourth section, our main results are stated and proved, which involves a lemma for estimating solutions to a class of SWEs and a Gronwall-type inequality over cones. A numerical illustration of the results is provided in Section 5.

2. Formulation of the problem. Let $(\Omega, \mathcal{F}, P)$ be a complete probability space and $W: \mathbb{R}_{+} \times \mathbb{R} \rightarrow L^{2}(\Omega)$, with $\mathbb{R}_{+}=[0, \infty)$, be a two-parameter Wiener process, which is understood in the Walsh setting [29]. Recall that $L^{2}(\Omega)$ is the space of all random variables $Y: \Omega \rightarrow \mathbb{R}$ such that $\mathbb{E}\left[Y^{2}\right]<\infty$. For $(t, x) \in \mathbb{R}_{+} \times \mathbb{R}$, let $C_{t, x}$ be the wave cone with vertex $(t, x)$, that is, the triangle delimited by the points $(t, x),(0, x+t),(0, x-t)$ :

$$
C_{t, x}=\{(s, y): 0 \leq s \leq t,|y-x| \leq t-s\} .
$$

We say that a function $\Psi: \mathbb{R}_{+} \times \mathbb{R} \rightarrow \mathbb{R}$ is increasing with respect to cones if it holds that $\Psi(s, y) \leq \Psi(t, x)$ for all $(s, y) \in C_{t, x}$. This means that

$$
C_{s, y} \subset C_{t, x} \Rightarrow \Psi(s, y) \leq \Psi(t, x) .
$$

The filtration $\left(\mathcal{F}_{C_{t, x}},(t, x) \in \mathbb{R}_{+} \times \mathbb{R}\right)$ is understood as follows: $\mathcal{F}_{C_{s, y}} \subset \mathcal{F}_{C_{t, x}}$ if $C_{s, y} \subset C_{t, x}$. A process $u: \mathbb{R}_{+} \times \mathbb{R} \rightarrow L^{2}(\Omega)$ is adapted if all random variables $u(t, x)$ are 
measurable with respect to the $\sigma$-field $\mathcal{F}_{C_{t, x}}$ for $(t, x) \in \mathbb{R}_{+} \times \mathbb{R}$. Moreover, for $t, s \in \mathbb{R}_{+}$, $x, y \in \mathbb{R}$, and every bounded, $\mathcal{F}_{C_{t, x}}$-measurable random variable $Y$, it holds that

$$
\mathbb{E}\left[Y \mid \mathcal{F}_{C_{s, y}}\right]=\mathbb{E}\left[Y \mid \mathcal{F}_{C_{s, y} \cap C_{t, x}}\right] .
$$

The filtration generated by rectangles (instead of cones) and satisfying (2.1) is said to be commuting $[12,14]$. Let $\mathcal{C}\left(C_{t, x}\right)$ be the space of continuous functions from $C_{t, x}$ to $\mathbb{R}$. By $\mathcal{X}$ we denote the space of all pathwise continuous and adapted processes $u: \mathbb{R}_{+} \times \mathbb{R} \rightarrow L^{2}(\Omega)$ with the seminorms

$$
\|u\|_{t, x}^{2}=\mathbb{E}\left[\sup _{(s, y) \in C_{t, x}}|u(t, x)|^{2}\right]<\infty \quad \text { for } t \in \mathbb{R}_{+}, x \in \mathbb{R} .
$$

Let $\mathcal{X}_{t, x}=\left\{\left.u\right|_{C_{t, x}}: u \in \mathcal{X}\right\}$. Consider the following initial value problem for the nonlinear stochastic wave equation (first introduced in [2]) with a non-local dependence

$$
\left\{\begin{aligned}
\frac{\partial^{2} u}{\partial t^{2}}-\frac{\partial^{2} u}{\partial x^{2}} & =f\left(t, x,\left.u\right|_{C_{t, x}}\right)+g\left(t, x,\left.u\right|_{C_{t, x}}\right) \dot{W} & & \text { for }(t, x) \in \mathbb{R}_{+} \times \mathbb{R} \\
u(0, x) & =\phi(x), \quad \frac{\partial u}{\partial t}(0, x)=\psi(x) & & \text { for } x \in \mathbb{R}
\end{aligned}\right.
$$

where $\dot{W}$ is white noise on $\mathbb{R}_{+} \times \mathbb{R}$ and $\phi(x), \psi(x)$ are deterministic and continuous functions. A process $u: \mathbb{R}_{+} \times \mathbb{R} \rightarrow L^{2}(\Omega)$ is a solution to (2.2) if $u \in \mathcal{X}$ and it satisfies the integral equation

$$
\begin{aligned}
u(t, x)= & \frac{1}{2}(\phi(x-t)+\phi(x+t))+\frac{1}{2} \int_{x-t}^{x+t} \psi(y) d y \\
& +\frac{1}{2} \int_{C_{t, x}} f\left(s, y,\left.u\right|_{C_{s, y}}\right) d y d s+\frac{1}{2} \int_{C_{t, x}} g\left(s, y,\left.u\right|_{C_{s, y}}\right) W(d s, d y),
\end{aligned}
$$

which is based on d'Alembert's formula corresponding to (2.2) and where the stochastic integral is interpreted in the sense of Walsh [29]. The existence and uniqueness of solutions to $(2.2)$ can be shown similarly as in [30].

3. The leap-frog scheme. Fix $h>0, t_{i}=i h, x_{j}=j h$ for $i \in \mathbb{N}, j \in \mathbb{Z}$, $\mathbb{N}=\{0,1,2, \ldots\}$. Set $E_{h}=\left\{\left(t_{i}, x_{j}\right) \in h \mathbb{N} \times h \mathbb{Z}, i+j\right.$ even $\}, u_{i, j}=u\left(t_{i}, x_{j}\right)$, for $\left(t_{i}, x_{j}\right) \in E_{h}$. We write $E_{h}^{\prime}$ for the complement of $E_{h}$. By $\Delta_{i, j}$ we denote the square (diamond) with center $\left(t_{i}, x_{j}\right)$ and corners at $\left(t_{i+1}, x_{j}\right),\left(t_{i-1}, x_{j}\right),\left(t_{i}, x_{j+1}\right),\left(t_{i}, x_{j-1}\right)$. Let $\Delta_{i, j}^{-}$, for $(i, j) \in \mathbb{N} \times \mathbb{Z}, i>0$, consist of all interior points of the square $\Delta_{i, j}$ and its two sides determined by the points $\left(t_{i}, x_{j+1}\right),\left(t_{i-1}, x_{j}\right)$ and $\left(t_{i}, x_{j-1}\right),\left(t_{i-1}, x_{j}\right)$, respectively, excluding these points. Let $\Delta_{0, j}^{-}$be the interior of the triangle with corners at $\left(t_{1}, x_{j}\right)$, $\left(0, x_{j-1}\right),\left(0, x_{j+1}\right)$ and the side determined by, but without, the points $\left(0, x_{j-1}\right),\left(0, x_{j+1}\right)$. The leap-frog method for (2.2) is of the form

$$
\left\{\begin{array}{rlr}
u_{i+1, j}= & u_{i, j+1}+u_{i, j-1}-u_{i-1, j}+h^{2} f\left(t_{i}, x_{j},\left.\left(I_{h} u\right)\right|_{C_{t_{i}, x_{j}}}\right) & \\
& +\frac{1}{2} g\left(t_{i}, x_{j},\left.\left(I_{h} u\right)\right|_{C_{t_{i}, x_{j}}}\right) W\left(\Delta_{i, j}\right), \quad\left(t_{i}, x_{j}\right) \in E_{h}^{\prime}, & \\
u_{0, j}= & \phi\left(x_{j}\right) & \\
u_{1, j}= & \frac{\phi\left(x_{j+1}\right)+\phi\left(x_{j-1}\right)}{2}+h \psi\left(x_{j}\right)+\frac{h^{2}}{2} f\left(0, x_{j},\left.\left(I_{h} u\right)\right|_{C_{0, x_{j}}}\right) & \\
& +\frac{1}{2} g\left(0, x_{j},\left.\left(I_{h} u\right)\right|_{C_{0, x_{j}}}\right) W\left(\Delta_{0, j}\right), & j \text { odd },
\end{array}\right.
$$


where $I_{h} u$ is the interpolating step function given by

$$
\left(I_{h} u\right)(t, x)= \begin{cases}\frac{1}{2}\left(u_{i, j-1}+u_{i, j+1}\right) & \text { for }(t, x) \in \Delta_{i, j}^{-}, \\ u_{i, j} & \text { for } t=t_{i}, x=x_{j},\left(t_{i}, x_{j}\right) \in E_{h} .\end{cases}
$$

Let $C_{i, j}^{h}=C_{t_{i}, x_{j}} \cap E_{h}$ and $\widetilde{C}_{i, j}^{h}=C_{t_{i}, x_{j}} \cap E_{h}^{\prime}$. For $\left(t_{i}, x_{j}\right) \in E_{h}$, we have

$$
\begin{aligned}
u_{i, j}=\frac{\phi\left(x_{j+i}\right)+\phi\left(x_{j-i}\right)}{2}+h \sum_{\substack{l:: \widetilde{C}_{i, j}^{h} \\
\left(0, x_{l}\right) \in}} \psi\left(x_{l}\right) \\
\quad+h^{2} \sum_{\substack{k, l: \\
\left(t_{k}, x_{l}\right) \in \widetilde{C}_{i, j}^{h}}} f\left(t_{k}, x_{l},\left.\left(I_{h} u\right)\right|_{C_{t_{k}, x_{l}}}\right)+\frac{1}{2} \sum_{\substack{k, l: \\
\left(t_{k}, x_{l}\right) \in \widetilde{C}_{i, j}^{h}}} g\left(t_{k}, x_{l},\left.\left(I_{h} u\right)\right|_{C_{t_{k}, x_{l}}}\right) W\left(\Delta_{k, l}\right) .
\end{aligned}
$$

Let $\mathcal{X}^{h}$ be the space of all discrete adapted processes $u: E_{h} \rightarrow L^{2}(\Omega)$ with the seminorms

$$
\|u\|_{i, j}^{2}=\mathbb{E}\left[\sup _{\substack{m, n: \\\left(t_{m}, x_{n}\right) \in C_{i, j}^{h}}}\left|u_{m, n}\right|^{2}\right]<\infty .
$$

For brevity, we write $\|u\|_{i, j}^{2}=\mathbb{E}\left[\sup _{C_{i, j}^{h}}\left|u_{m, n}\right|^{2}\right]$.

4. Stability of the leap-frog method. Before stating the main result, we formulate the following lemma for estimating solutions to a class of SWEs.

Lemma 4.1. Suppose that $\alpha_{i, j}^{(1)}, \alpha_{i, j}^{(2)} \in \mathcal{X}^{h}$, for $i, j:\left(t_{i}, x_{j}\right) \in E_{h}$. Let a process $\varepsilon_{i, j} \in \mathcal{X}^{h}$ satisfy the following equation

$$
\left\{\begin{array}{rlrl}
\varepsilon_{i+1, j} & =\varepsilon_{i, j+1}+\varepsilon_{i, j-1}-\varepsilon_{i-1, j}+h^{2} \alpha_{i, j}^{(1)}+\frac{1}{2} \alpha_{i, j}^{(2)} W\left(\Delta_{i, j}\right), & \left(t_{i}, x_{j}\right) \in E_{h}^{\prime} \\
\varepsilon_{0, j} & =0 & & j \text { even }, \\
\varepsilon_{1, j} & =\frac{h^{2}}{2} \alpha_{0, j}^{(1)}+\alpha_{0, j}^{(2)} W\left(\Delta_{0, j}\right), & & j \text { odd } .
\end{array}\right.
$$

Then there exists a nonnegative constant $K$ such that

$$
\|\varepsilon\|_{i, j}^{2} \leq 2 i^{2} h^{4} \sum_{\widetilde{C}_{i, j}^{h}}\left\|\alpha^{(1)}\right\|_{m, n}^{2}+4 K h^{2} \sum_{\widetilde{C}_{i, j}^{h}}\left\|\alpha^{(2)}\right\|_{m, n}^{2}
$$

for $i, j:\left(t_{i}, x_{j}\right) \in E_{h}$.

Proof. The recursive use of (4.1) leads to

$$
\varepsilon_{i, j}=h^{2} \sum_{\widetilde{C}_{i, j}^{h}} \alpha_{k, l}^{(1)}+\sum_{\widetilde{C}_{i, j}^{h}} \alpha_{k, l}^{(2)} W\left(\Delta_{k, l}\right) \quad \text { for }\left(t_{i}, x_{j}\right) \in E_{h} .
$$

The elementary inequality $(a+b)^{2} \leq 2 a^{2}+2 b^{2}$ yields

$$
\begin{aligned}
\mathbb{E}\left[\sup _{C_{i, j}^{h}}\left|\varepsilon_{m, n}\right|^{2}\right] & \leq 2 h^{4} \mathbb{E}\left[\sup _{C_{i, j}^{h}}\left|\sum_{\widetilde{C}_{m, n}^{h}} \alpha_{k, l}^{(1)}\right|^{2}\right]+2 \mathbb{E}\left[\sup _{C_{i, j}^{h}}\left|\sum_{\widetilde{C}_{m, n}^{h}} \alpha_{k, l}^{(2)} W\left(\Delta_{k, l}\right)\right|^{2}\right] \\
& =: 2 h^{4} J_{i, j}^{(1)}+2 J_{i, j}^{(2)} .
\end{aligned}
$$


Applying the Schwarz inequality we have

$$
J_{i, j}^{(1)} \leq\left|\widetilde{C}_{i, j}^{h}\right| \mathbb{E}\left[\sup _{C_{i, j}^{h}} \sum_{\widetilde{C}_{m, n}^{h}}\left(\alpha_{k, l}^{(1)}\right)^{2}\right] \leq i^{2} \sum_{\widetilde{C}_{i, j}^{h}} \mathbb{E}\left[\sup _{C_{m, n}^{h}}\left(\alpha_{k, l}^{(1)}\right)^{2}\right]=i^{2} \sum_{\widetilde{C}_{i, j}^{h}}\left\|\alpha^{(1)}\right\|_{m, n}^{2} .
$$

By the Cairoli inequality $[13,12]$, there is a nonnegative constant $K$ such that

$$
J_{i, j}^{(2)} \leq K\left|\Delta_{i, j}\right| \mathbb{E}\left[\sum_{\widetilde{C}_{i, j}^{h}}\left(\alpha_{k, l}^{(2)}\right)^{2}\right] \leq 2 K h^{2} \sum_{\widetilde{C}_{i, j}^{h}} \mathbb{E}\left[\sup _{C_{m, n}^{h}}\left(\alpha_{k, l}^{(2)}\right)^{2}\right] \leq 2 K h^{2} \sum_{\widetilde{C}_{i, j}^{h}}\left\|\alpha^{(2)}\right\|_{m, n}^{2} .
$$

Hence, the proof is complete.

We need the following inequality (cf. [23, Theorem 2.1.56]).

LEMMA 4.2. Let $V_{i}$ be a real-valued non-negative function defined on $\mathbb{Z}_{\gamma, \delta}=\{i \in \mathbb{Z}, \gamma \leq i \leq \delta, \gamma, \delta \in \mathbb{Z}\}, E=\left\{(i, j) \in \mathbb{Z}^{2}: \gamma \leq j \leq i \leq \delta\right\}$. Let $c_{i, j}$, $d_{i, j}$ be real-valued non-negative functions defined on $E$ and non-decreasing in $i$ for each $j \in \mathbb{Z}_{\gamma, \delta}$, and suppose that for all $i \in \mathbb{Z}_{\gamma, \delta}$,

$$
V_{i} \leq c+\sum_{m=\gamma}^{i-1} c_{i, m} V_{m}+\sum_{m=\gamma}^{\delta} d_{i, m} V_{m}
$$

where $c \geq 0$ is a constant. If

$$
r_{i}=\sum_{j=\gamma}^{\delta} d_{i, j} \prod_{m=\gamma}^{j-1}\left(1+c_{j, m}\right)<1
$$

then for all $i \in \mathbb{Z}_{\gamma, \delta}$,

$$
V_{i} \leq \frac{c}{1-r_{i}} \prod_{m=\gamma}^{i-1}\left(1+c_{i, m}\right) .
$$

We formulate the following Gronwall-type inequality over cones. The proof is based on taking suprema of the space variable and applying Lemma 4.2.

LEMMA 4.3. Let $v_{i, j}, a_{i, j}, b_{i, j}$ be nonnegative functions, for $i \in \mathbb{N}, j \in \mathbb{Z}$, and let $a_{i, j}$, $b_{i, j}$ be increasing with respect to cones. If

$$
v_{i, j} \leq a_{i, j}+b_{i, j} \sum_{C_{i, j}^{h}} v_{m, n}
$$

for $i, j:\left(t_{i}, x_{j}\right) \in E_{h}$, then

$$
v_{i, j} \leq a_{i, j} \prod_{m=0}^{i-1}\left(1+[2(i-m)+1] b_{i, j}\right)
$$

for $i, j:\left(t_{i}, x_{j}\right) \in E_{h}$.

Proof. Fix $h>0,\left(t_{i_{0}}, x_{j_{0}}\right) \in E_{h}$. Since $a_{i, j}, b_{i, j}$ are increasing with respect to cones, we have

$$
v_{i, j} \leq a_{i_{0}, j_{0}}+b_{i_{0}, j_{0}} \sum_{m=0}^{i-1} \sum_{n=j-i+m}^{j+i-m} v_{m, n}
$$


for $i, j:\left(t_{i}, x_{j}\right) \in C_{i_{0}, j_{0}}^{h}$. If we define

$$
V_{i}=\sup _{\substack{j \in \mathbb{Z} \\\left|j-j_{0}\right| \leq i_{0}-i}} v_{i, j}, \quad i=0,1, \ldots, i_{0}
$$

then we get

$$
v_{i, j} \leq a_{i_{0}, j_{0}}+b_{i_{0}, j_{0}} \sum_{m=0}^{i-1}[2(i-m)+1] V_{m} .
$$

Hence,

$$
V_{i} \leq a_{i_{0}, j_{0}}+b_{i_{0}, j_{0}} \sum_{m=0}^{i-1}[2(i-m)+1] V_{m} .
$$

Applying Lemma 4.2 with $c=a_{i_{0}, j_{0}}, c_{i, j}=b_{i_{0}, j_{0}}(2(i-j)+1), d_{i, j}=0$, we obtain

$$
V_{i} \leq a_{i_{0}, j_{0}} \prod_{m=0}^{i-1}\left(1+[2(i-m)+1] b_{i_{0}, j_{0}}\right)
$$

Since $v_{i, j} \leq V_{i}$, for $i, j:\left(t_{i}, x_{j}\right) \in C_{i_{0}, j_{0}}^{h}$ and arbitrary $\left(t_{i_{0}}, x_{j_{0}}\right) \in E_{h}$, the proof is complete.

By the stability of the scheme we mean that small perturbations to the scheme lead to small changes in the solution of a differential equation, i.e., $\lim _{h \rightarrow 0}\|\bar{u}-u\|_{i, j}^{2}=0$, where $u$, $\bar{u}$ are the solutions to (3.1) and its perturbation, respectively, and the perturbation functions $\xi$, $\eta$ satisfy $\lim _{h \rightarrow 0} \sup _{i \in \mathbb{N}, j \in \mathbb{Z}}\left(\|\xi\|_{i, j}+\|\eta\|_{i, j}\right)=0$.

THEOREM 4.4. Suppose that the functions $(t, x) \mapsto f(t, x, 0),(t, x) \mapsto g(t, x, 0)$ are continuous on $\mathbb{R}_{+} \times \mathbb{R}$ such that

$$
|f(t, x, 0)|+|g(t, x, 0)| \leq M(t, x)
$$

and the functions $f(t, x, \cdot), g(t, x, \cdot)$ satisfy the Lipschitz condition

$$
\begin{aligned}
& \left|f\left(t, x,\left.u\right|_{C_{t, x}}\right)-f\left(t, x,\left.\bar{u}\right|_{C_{t, x}}\right)\right| \leq L(t, x) \sup _{(s, y) \in C_{t, x}}|u(s, y)-\bar{u}(s, y)|, \\
& \left|g\left(t, x,\left.u\right|_{C_{t, x}}\right)-g\left(t, x,\left.\bar{u}\right|_{C_{t, x}}\right)\right| \leq L(t, x) \sup _{(s, y) \in C_{t, x}}|u(s, y)-\bar{u}(s, y)|,
\end{aligned}
$$

where $M, L:[0, T] \times \mathbb{R} \rightarrow \mathbb{R}_{+}$are increasing with respect to cones. Then the leap-frog method (3.1) for (2.2) is stable.

Proof. Fix $h>0,\left(t_{i_{0}}, x_{j_{0}}\right) \in E_{h}$. Let $u_{i, j}$ be the solution to (3.1) and $\bar{u}_{i, j}$ the solution to the perturbed scheme

$$
\left\{\begin{array}{c}
\left.\bar{u}_{i+1, j}=\bar{u}_{i, j+1}+\bar{u}_{i, j-1}-\bar{u}_{i-1, j}+h^{2} f\left(t_{i}, x_{j},\left.\left(I_{h} \bar{u}\right)\right|_{C_{t_{i}, x_{j}}}\right)\right)+h^{2} \xi_{i, j} \\
\left.\quad+\frac{1}{2} g\left(t_{i}, x_{j},\left.\left(I_{h} \bar{u}\right)\right|_{C_{t_{i}, x_{j}}}\right)\right) W\left(\Delta_{i, j}\right)+\frac{1}{2} \eta_{i, j} W\left(\Delta_{i, j}\right), \quad\left(t_{i}, x_{j}\right) \in E_{h}^{\prime}, \\
\bar{u}_{0, j}=\phi\left(x_{j}\right) \\
\bar{u}_{1, j}=\frac{\phi\left(x_{j+1}\right)+\phi\left(x_{j-1}\right)}{2}+h \psi\left(x_{j}\right)+\frac{h^{2}}{2} f\left(0, x_{j},\left.\left(I_{h} \bar{u}\right)\right|_{C_{0, x_{j}}}\right) \\
\quad+\frac{1}{2} g\left(0, x_{j},\left.\left(I_{h} \bar{u}\right)\right|_{C_{0, x_{j}}}\right) W\left(\Delta_{0, j}\right), \quad j \text { odd },
\end{array}\right.
$$


where $\xi_{i, j}, \eta_{i, j} \in \mathcal{X}^{h}$, for $i, j:\left(t_{i}, x_{j}\right) \in \widetilde{C}_{i_{0}, j_{0}}^{h}$, are such that

$$
\lim _{h \rightarrow 0} \sup _{i \in \mathbb{N}, j \in \mathbb{Z}}\left(\|\xi\|_{i, j}+\|\eta\|_{i, j}\right)=0 .
$$

In order to apply Lemma 4.1, we take $\varepsilon_{i, j}=\bar{u}_{i, j}-u_{i, j}$ and

$$
\begin{aligned}
& \alpha_{i, j}^{(1)}=f\left(t_{i}, x_{j},\left.\left(I_{h} \bar{u}\right)\right|_{C_{t_{i}, x_{j}}}\right)-f\left(t_{i}, x_{j},\left.\left(I_{h} u\right)\right|_{C_{t_{i}, x_{j}}}\right)+\xi_{i, j}, \\
& \alpha_{i, j}^{(2)}=g\left(t_{i}, x_{j},\left.\left(I_{h} \bar{u}\right)\right|_{C_{t_{i}, x_{j}}}\right)-g\left(t_{i}, x_{j},\left.\left(I_{h} u\right)\right|_{C_{t_{i}, x_{j}}}\right)+\eta_{i, j} .
\end{aligned}
$$

Next, by the Lipschitz condition (4.3) for $f$, we have

$$
\begin{aligned}
\mathbb{E}\left[\sup _{C_{m, n}^{h}}\left|\alpha_{k, l}^{(1)}\right|^{2}\right] & =\mathbb{E}\left[\sup _{C_{m, n}^{h}}\left|f\left(t_{k}, x_{l},\left.\left(I_{h} \bar{u}\right)\right|_{C_{t_{k}, x_{l}}}\right)-f\left(t_{k}, x_{l},\left.\left(I_{h} u\right)\right|_{C_{t_{k}, x_{l}}}\right)+\xi_{k, l}\right|^{2}\right] \\
& \leq 2 L^{2}\left(t_{m}, x_{n}\right)\|\bar{u}-u\|_{m, n}^{2}+2\|\xi\|_{m, n}^{2} .
\end{aligned}
$$

Hence

$$
\left\|\alpha^{(1)}\right\|_{m, n}^{2} \leq 2 L^{2}\left(t_{m}, x_{n}\right)\|\bar{u}-u\|_{m, n}^{2}+2\|\xi\|_{m, n}^{2} .
$$

Similarly,

$$
\left\|\alpha^{(2)}\right\|_{m, n}^{2} \leq 2 L^{2}\left(t_{m}, x_{n}\right)\|\bar{u}-u\|_{m, n}^{2}+2\|\eta\|_{m, n}^{2} .
$$

Thus, we have the estimate

$$
\begin{aligned}
\|\bar{u}-u\|_{i, j}^{2} \leq 4 i^{2} h^{2}\left(i^{2} h^{2}\|\xi\|_{i, j}^{2}+2 K\|\eta\|_{i, j}^{2}\right) & \\
& +4 h^{2} L^{2}\left(t_{i}, x_{j}\right)\left(i^{2} h^{2}+2 K\right) \sum_{\widetilde{C}_{i, j}^{h}}\|\bar{u}-u\|_{m, n}^{2}
\end{aligned}
$$

for some $K>0$. By Lemma 4.3 we obtain

$$
\|\bar{u}-u\|_{i, j}^{2} \leq C_{1} \prod_{m=0}^{i-1}\left(1+[2(i-m)+1] C_{2}\right)
$$

for $i, j:\left(t_{i}, x_{j}\right) \in C_{i_{0}, j_{0}}^{h}$ and

$$
C_{1}=4 i^{2} h^{2}\left(i_{0}^{2} h^{2}\|\xi\|_{i_{0}, j_{0}}^{2}+2 K\|\eta\|_{i_{0}, j_{0}}^{2}\right), \quad C_{2}=4 h^{2} L^{2}\left(t_{i_{0}}, x_{j_{0}}\right)\left(i_{0}^{2} h^{2}+2 K\right) .
$$

Since for arbitrary $\left(t_{i_{0}}, x_{j_{0}}\right) \in E_{h}$ we have $\|\bar{u}-u\|_{i_{0}, j_{0}}^{2} \rightarrow 0$ as $h \rightarrow 0$ provided (4.4) holds, the proof is complete.

REMARK 4.5. Conditions (4.2), (4.3) imply a linear growth of $f$ and $g$.

REMARK 4.6. The leap-frog scheme (3.1) converges in $L^{2}$ at a rate of $O(\sqrt{h})$, which is the same as the rate obtained in [30]. Indeed, the norm of $u_{i, j}-u\left(t_{i}, x_{j}\right)$, where $u$ is given by (2.3) at $\left(t_{i}, x_{j}\right)$ and $u_{i, j}$ is given by (3.2), can be estimated by the sum of two functions of the orders $O(h)$ and $O(\sqrt{h})$. 
5. Numerical experiments. In [28] the time-space white noise with rectangular increments is discretised at $\left(t_{i}, x_{j}\right)$ as follows

$$
\frac{\partial^{2} W}{\partial t \partial x} \approx \frac{1}{\sqrt{\Delta t \Delta x}} N_{i, j},
$$

where $N_{i, j}$ are independent standard (zero mean, unit variance) normal random variables and $\Delta t, \Delta x$ are time and space step sizes, respectively. Due to the conical nature of problem (2.2), we propose the following generation of the two-parameter Brownian motion over cones. Fix $h>0,\left(t_{i_{0}}, x_{j_{0}}\right) \in E_{h}$, and let

$$
\widehat{C}_{i_{0}, j_{0}}^{h}:=C_{i_{0}, j_{0}} \cap\left(h \mathbb{N}_{+} \times h \mathbb{Z}\right), \quad \mathbb{N}_{+}=\{1,2, \ldots\} .
$$

Suppose we have a sequence $\left\{Y_{k, l},(k h, l h) \in \widehat{C}_{i_{0}, j_{0}}^{h} \cap E_{h}^{\prime}\right\}$ of independent standard normal random variables indexed by the centers of the diamonds. The random variables $Y_{k, l}$ will be generated by a computer random number generator. We define the Brownian motion over a diamond with vertices $((i-2) h, j h),(i h, j h),((i-1) h,(j+1) h),((i-1) h,(j-1) h)$ by

$$
W\left(\Delta_{i-1, j}^{h}\right)= \begin{cases}\sqrt{2} h Y_{i-1, j}, & i \geq 2,(i h, j h) \in C_{i_{0}, j_{0}} \cap E_{h} \\ h Y_{i-1, j}, & i=1,(h, j h) \in C_{i_{0}, j_{0}} \cap E_{h}\end{cases}
$$

Notice that (5.1) has the desired properties $\mathbb{E}\left[W\left(\Delta_{i-1, j}^{h}\right)\right]=0$ and

$$
\operatorname{Var}\left[W\left(\Delta_{i-1, j}^{h}\right)\right]= \begin{cases}2 h^{2}, & i \geq 2,(i h, j h) \in C_{i_{0}, j_{0}} \cap E_{h}, \\ h^{2}, & j \in \mathbb{Z},(h, j h) \in C_{i_{0}, j_{0}} \cap E_{h} .\end{cases}
$$

Now consider the diamond $\Delta_{i-2, j}^{2 h}$ composed of four diamonds $\Delta_{i-3, j}^{h}, \Delta_{i-2, j+1}^{h}, \Delta_{i-2, j-1}^{h}$, $\Delta_{i-1, j}^{h}$. Suppose we have defined $W\left(\Delta_{i-3, j}^{h}\right), W\left(\Delta_{i-2, j+1}^{h}\right), W\left(\Delta_{i-2, j-1}^{h}\right), W\left(\Delta_{i-1, j}^{h}\right)$. We define the Brownian motion $W\left(\Delta_{i-2, j}^{2 h}\right)$ over the diamond $\Delta_{i-2, j}^{2 h}$ on the grid with the step size $2 h$ as follows:

$$
W\left(\Delta_{i-2, j}^{2 h}\right)=\left\{\begin{array}{c}
W\left(\Delta_{i-3, j}^{h}\right)+W\left(\Delta_{i-2, j+1}^{h}\right)+W\left(\Delta_{i-2, j-1}^{h}\right)+W\left(\Delta_{i-1, j}^{h}\right), \\
i \geq 4,(i h, j h) \in C_{i_{0}, j_{0}} \cap E_{2 h} \\
W\left(\Delta_{0, j+1}^{h}\right)+W\left(\Delta_{0, j-1}^{h}\right)+W\left(\Delta_{1, j}^{h}\right) \\
i=2,(2 h, 2 j h) \in C_{i_{0}, j_{0}} \cap E_{2 h} .
\end{array}\right.
$$

Notice that (5.2) has the desired properties $\mathbb{E}\left[W\left(\Delta_{i-2, j}^{2 h}\right)\right]=0$ and

$$
\operatorname{Var}\left[W\left(\Delta_{i-2, j}^{2 h}\right)\right]= \begin{cases}2 \cdot 4 h^{2}, & i \geq 4,(i h, j h) \in C_{i_{0}, j_{0}} \cap E_{2 h}, \\ 4 h^{2}, & j \in \mathbb{Z},(2 h, j h) \in C_{i_{0}, j_{0}} \cap E_{2 h} .\end{cases}
$$

Figure 5.1 displays the solution of the leap-frog method (3.1) for the deterministic problem of the form

$$
\begin{array}{ll}
f\left(t, x,\left.u\right|_{C_{t, x}}\right)=0, & g\left(t, x,\left.u\right|_{C_{t, x}}\right)=0, \\
\varphi(x)=\cos (x), & \psi(x)=-2 \sin (x) .
\end{array}
$$




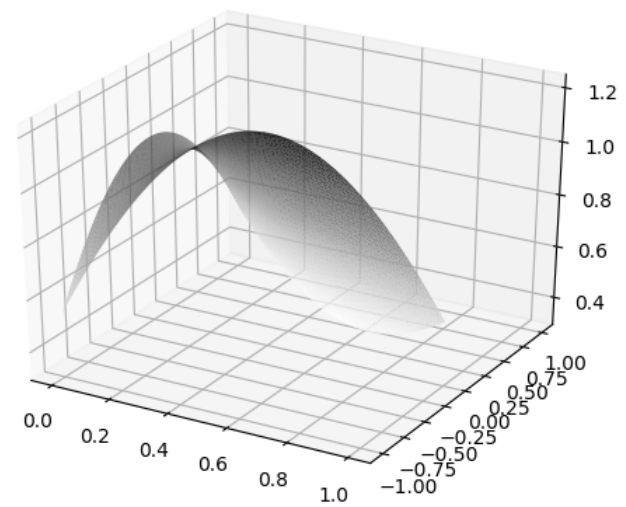

FIG. 5.1. Numerical solution for the deterministic problem, $h=0.01$.

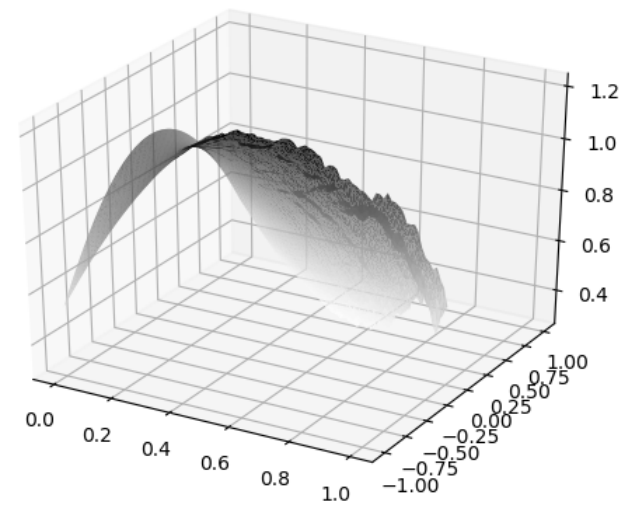

FIG. 5.2. Numerical solution for the stochastic problem, $h=0.01$.

Replacing the zero function $g$ in (5.3) with

$$
g\left(t, x,\left.u\right|_{C_{t, x}}\right)=\sin \left(\int_{C_{t, x}} u(s, y) d y d s\right)
$$

and including the noise term yields the leap-frog solution displayed in Figure 5.2. Tables 5.1 and 5.2 describe the dependence of the numerical solution on the grid size $h$ and the number of sample paths. 


\section{ETNA}

Kent State University and

Johann Radon Institute (RICAM)

TABLE 5.1

The errors for the numerical solution for 50 sample paths.

\begin{tabular}{c|c}
$h$ & $\mathbb{E}\left[\sup \left|u_{h}-u_{2 h}\right|^{2}\right]$ \\
\hline 0.02 & $6.490137 \cdot 10^{-4}$ \\
0.01 & $2.281072 \cdot 10^{-4}$
\end{tabular}

TABLE 5.2

The errors for the numerical solution for 100 sample paths.

\begin{tabular}{c|c}
$h$ & $\mathbb{E}\left[\sup \left|u_{h}-u_{2 h}\right|^{2}\right]$ \\
\hline 0.02 & $5.627765 \cdot 10^{-4}$ \\
0.01 & $2.185586 \cdot 10^{-4}$
\end{tabular}

\section{REFERENCES}

[1] C. Benham, S. Harvey, W. Olson, D. W. Sumners, and D. Swigon, eds., Mathematics of DNA Structure, Function and Interactions, Springer, Dordrecht, 2009.

[2] E. CABAÑA, The vibrating string forced by white noise, Z. Wahrscheinlichkeitstheorie und Verw. Gebiete, 15 (1970), pp. 111-130.

[3] D. Cohen, S. LARsson, And M. SigG, A trigonometric method for the linear stochastic wave equation, SIAM J. Numer. Anal., 51 (2013), pp. 204-222.

[4] R. C. DAlang, The stochastic wave equation, in A Minicourse on Stochastic Partial Differential Equations, D. Khoshnevisan and F. Rassoul-Agha, eds., Lect. Notes in Math., vol. 1962, Springer, Berlin, 2009, pp. 39-71.

[5] V. Y. Glotov, V. M. Goloviznin, S. A. Karabasov, and A. P. Markesteijn, New two-level leapfrog scheme for modeling the stochastic Landau-Lifshitz equations, Comput. Math. Math. Phys., 54 (2014), pp. 315-334.

[6] O. GonZALEZ AND J. H. MADDOCKS, Extracting parameters for base-pair level models of DNA frommolecular dynamics simulations, Theor. Chem. Acc., 106 (2001), pp. 76-82.

[7] E. HAIRER, C. LUBICH, AND G. WANNER, Geometric numerical integration illustrated by the Störmer-Verlet method, Acta Numer., 12 (2003), pp. 399-450.

[8] E. Hausenblas, Weak approximation of the stochastic wave equation, J. Comput. Appl. Math., 235 (2010), pp. 33-58.

[9] A. Hellemans, Soho probes sun's interior by tuning in to its vibrations, Science, 272 (1996), pp. 1264-1265.

[10] A. IsERLES, A First Course in the Numerical Analysis of Differential Equations, Cambridge University Press, Cambridge, 1996.

[11] N. JiAng, M. KubaCki, W. LAYton, M. Moraiti, And H. Tran, A Crank-Nicolson Leapfrog stabilization: unconditional stability and two applications, J. Comput. Appl. Math., 281 (2015), pp. 263-276.

[12] D. Khoshnevis An, Multiparameter Processes, Springer, New York, 2002.

[13] O. KLESOV, Limit Theorems for Multi-Indexed Sums of Random Variables, Springer, Heidelberg, 2014.

[14] P. S. Knopov And O. N. Deriyeva, Estimation and Control Problems for Stochastic Partial Differential Equations, Springer, New York, 2013.

[15] M. Kovács, S. LARSSON, AND F. SAEDPANAH, Finite element approximation of the linear stochastic wave equation with additive noise, SIAM J. Numer. Anal., 48 (2010), pp. 408-427.

[16] H. LESZCZYŃSKI AND M. WRZOSEK, Newton's method for nonlinear stochastic wave equations driven by one-dimensional Brownian motion, Math. Biosci. Eng., 14 (2017), pp. 237-248.

[17] J. Li, J. WANG WATERS, AND E. A. MACHORRo, An implicit leap-frog discontinuous Galerkin method for the time-domain Maxwell's equations in metamaterials, Comput. Methods Appl. Mech. Engrg., 223/224 (2012), pp. 43-54.

[18] J. LIU AND M. XIAO, A leapfrog multigrid algorithm for the optimal control of parabolic PDEs with Robin boundary conditions, J. Comput. Appl. Math., 307 (2016), pp. 216-234.

[19] A. Mahalov And M. Moustaoui, Time-filtered leapfrog integration of Maxwell equations using unstaggered temporal grids, J. Comput. Phys., 325 (2016), pp. 98-115.

[20] A. MARTIN, S. M. PRIGARIN, AND G. WINKLER, Exact and fast numerical algorithms for the stochastic wave equation, Int. J. Comput. Math., 80 (2003), pp. 1535-1541.

[21] L. NOAKES AND R. KOZERA, The 2-D leap-frog: integrability, noise, and digitization, in Digital and Image Geometry, G. Bertrand, A. Imiya, and R. Klette, eds., Lect. Notes in Comput. Sci., vol. 2243, Springer, Berlin, 2001, pp. 352-364.

[22] G. DA Prato And J. ZABCZYK, Stochastic equations in Infinite Dimensions, Cambridge University Press, Cambridge, 1992.

[23] Y. QIN, Integral and Discrete Inequalities and Their Applications. Vol. I: Linear Inequalities, Birkhäuser, Cham, 2016.

[24] L. QUER-SARDANYONS AND M. SAnZ-SolÉ, Space semi-discretisations for a stochastic wave equation, Potential Anal., 24 (2006), pp. 303-332.

[25] H. SCHURZ, A numerical method for nonlinear stochastic wave equations in $\mathbb{R}^{1}$, Dyn. Contin. Discrete Impuls. 
Syst. Ser. A Math. Anal., 14 (2007), pp. 74-78.

[26] A. SEPEHRI, A mathematical model for DNA, Int. J. Geom. Methods Mod. Phys., 14 (2017), Art. 1750152, 27 pages.

[27] W. SHI, X. WU, AND J. XIA, Explicit multi-symplectic extended leap-frog methods for Hamiltonian wave equations, J. Comput. Phys., 231 (2012), pp. 7671-7694.

[28] H. C. TUCKWELL, Analytical and simulation results for the stochastic spatial FitzHugh-Nagumo model neuron, Neural Comput., 20 (2008), pp. 3003-3033.

[29] J. B. WALSH, An introduction to stochastic partial differential equations, in École d'été de Probabilités de Saint-Flour, XIV_-1984, P. L. Hennequin, ed., Lect. Notes in Math., vol. 1180, Springer, Berlin, 1986, pp. 265-439.

[30] - On numerical solutions of the stochastic wave equation, Illinois J. Math., 50 (2006), pp. 991-1018.

[31] X. WANG, S. GAN, AND J. TANG, Higher order strong approximations of semilinear stochastic wave equation with additive space-time white noise, SIAM J. Sci. Comput., 36 (2014), pp. A2611-A2632. 